\title{
Antioxydant and Endotheliotropic Properties 4-Hydroxy-3,5-Di-Tret-Butyl Cinnamic Acid under Conditions of Experimental Brain Ischemia
}

\author{
Pozdnyakov DI", Voronkov AV, Zolotych DS, Arlt AV \\ Pyatigorsk Medical and Pharmaceutical Institute, A Branch of Volgograd State Medical University, Pyatigorsk, RUSSIA.
}

\begin{abstract}
Objective: To assess the effect of 4-hydroxy-3,5-di-tret-butyl cinnamic acid on the state of endothelial function under conditions of experimental cerebral ischemia. Materials and Methods: Antioxidant properties of 4-hydroxy-3,5-di-tret-butyl cinnamic acid and the effect of this compound on NO-synthase system activity and ADMA concentration were evaluated. Results: As a result, it has been found that the use of 4-hydroxy-3,5-di-tretbutyl cinnamic acid promotes reduction activity of catalase, glutathione peroxidase, superoxide dismutase and a decrease in the concentration of MDA and DC. In addition, the ability to modulate NO synthase system activity was established for this compound: an increase in eNOS activity by $114.2 \%(p<0.05)$, decrease activity of nNOS and iNOS by $60.6 \%(p<0.05)$ and $61.9 \%(p<0.05)$, respectively, decrease concentration of ADMA by $83.9 \%(p<0.05)$. Conclusion: The results of the conducted researches it
\end{abstract}

is established that 4-hydroxy-3,5-di-tret-butyl cinnamic acid has antioxidant and NOS - regulating effect.

Key words: Experimental ischemic stroke, Derivatives of cinnamic acid, Antioxidant, Endothelium, Endothelioprotection, Rats.

Correspondence

D.I. Pozdnyakov, Pyatigorsk Medical and Pharmaceutical Institute, A Branch of Volgograd State Medical University, Pyatigorsk, RUSSIA.

Phone: +7-918-756-08-89

Email: pozdniackow.dmitry@yandex.ru

DOI: 10.5530/jyp.2018.10.43

\section{INTRODUCTION}

Ischemic stroke is one of the leading causes of death and disability of the population, both in economically developed and developing countries. "Rejuvenation" of ischemic stroke makes this condition a huge medical and socioeconomic problem, since it affects a large part of the able-bodied and economically active population. ${ }^{1,2}$ In view of the heterogeneity of ischemic stroke, the complexity of its pathogenesis, it is not always possible to achieve the target level of reducing the consequences of this condition by traditional methods of early cerebroprotection, which include the pharmacotherapeutic effects of such groups of drugs as: NMDA antagonists, calcium channel blockers, growth factors, antioxidants etc., dictates the need to search for new "targets" for therapeutic action. ${ }^{3}$ In a number of experimental studies, it has been suggested that the preservation of endothelial function, can somewhat offset the consequences of ischemic stroke, and also enhance the pharmacological effect of traditional methods of stroke therapy-thrombolysis. ${ }^{4}$ However, to date, the spectrum of pharmacologically active compounds that can favorably affect vascular endothelial function is limited, and the effect on the endothelial function of biologically active substances often has an additional (pleiotropic) character. Among potential endothelioprotectors, as the most promising objects for study, it is possible to identify compounds that have antioxidant properties. ${ }^{5}$ Elimination of one of the leading factors of cell damage, including endothelial cells, oxidative stress, helps to normalize their functional activity, restore the production of vasoactive and antiplatelet / anticoagulant factors, and also reduces the biodegradation of the formed nitric oxide into peroxonitrite, thereby reducing the secondary a wave of oxidative damage to cellular structures - "nitrite stress," which also has a beneficial effect on endothelial function. ${ }^{6}$ In this connection, the search for substances capable of preserving the structural and functional integrity of endotheliocytes and possessing antioxidant properties is of indisputable scientific and practical interest, thus providing a much milder course of ischemic stroke, and also increasing the chances of a favorable outcome of the disease.?

\section{MATERIALS AND METHODS}

80 male Wistar rats weighing 200-220 grams were used in the experiment. All animal manipulations conformed to the norms of international experimental ethics (European Convention for the Protection of Vertebrates used for experimental and other scientific purposes (Strasbourg, 22 June 1998)). Experimental groups were formed by randomization of animals by age and weight. In each group there were 20 individuals. The first group of animals - pseudo-operated rats (PO), second - negative control (NC). Sulodexide (Wessel Doué F, Alfa Wasserman, Italy) at a dose $30 \mathrm{UIL},{ }^{8}$ ethylmethylhydroxypyridinosuccinate («Mexidol», PHARMASOFT) at a dose of $30 \mathrm{mg} / \mathrm{kg}^{9}$ and thioctic acid («Octolipen», Pharmstandard) in a dose of $50 \mathrm{mg} / \mathrm{kg}^{10}$ was used as a reference preparation: The test compound 4-hydroxy-3,5-di-tret-butyl cinnamic acid (ATACL) was administered at a dose of $100 \mathrm{mg} / \mathrm{kg}$. Comparison drugs and study compound were administered per os, after the reproduction of focal cerebral ischemia, and daily for 3 days.

\section{Experimental model of focal cerebral ischemia}

Focal ischemia was reproduced by a coagulation method under conditions of chloral hydrate $(350 \mathrm{mg} / \mathrm{kg})$ anesthesia. The animals were anesthetized, depilated a patch of skin below and to the left of the right eye, made an incision. Pulled soft tissues, removed the procession of the malar bone and uncovered the cranium at the intersection of the right 
middle cerebral artery with the olfactory tract. Drilled a trepanation hole with a diameter of $\approx 2 \mathrm{~cm}^{2}$, removed the dura mater and coagulated the middle cerebral artery, while under the magnifying glass observed the cessation of blood flow in the arterial bed. Then, bone fragments were repositioned, the wound was layer-by-layer, and treated with an antiseptic. ${ }^{11}$

\section{Determination of the concentration of diene conjugates}

Determination of the content of diene conjugates (DC) of acyl hydroperoxides of fatty acids in the brain homogenate was carried out spectrophotometrically at $233 \mathrm{~nm}$. The DC was extracted with a mixture of heptane: isopropanol (1: 1). The number of DCs was calculated from the molar extinction coefficient of conjugated dienes at $233 \mathrm{~nm} 2.2 \times 10$ $5 \mathrm{M}^{-1} \mathrm{~cm}^{-1}$ and expressed in $\mathrm{nmol} / \mathrm{mg}$ protein. ${ }^{12}$

\section{Determination of malonic dialdehyde concentration}

The content of malonic dialdehyde (MDA) was determined in the brain homogenate by a spectrophotometric method. This method is based on the formation of a colored reaction product of MDA with 2-thiobarbituric acid, having an absorption maximum at $532 \mathrm{~nm}$. The color of the solution is proportional to the concentration of malonic dialdehyde. The amount of MDA was calculated from the value of the molar extinction coefficient $\left(1.56^{*} 10^{5} \mathrm{~L} / \mathrm{mol}^{-1} / \mathrm{cm}^{-1}\right)$, the results were expressed in $\mathrm{nmol} / \mathrm{mg}$ protein.

\section{Determination of catalase activity}

The activity of catalase was determined in the brain ultracentrifuged by the spectrophotometric method for the rate of decomposition of hydrogen peroxide. The amount of hydrogen peroxide was determined in the reaction with $4 \%$ ammonium molybdate solution. The color intensity of the reaction product was measured at $410 \mathrm{~nm}$. The catalase activity was calculated from the difference between the extinctions of the experimental and idle samples, using the molar extinction coefficient of hydrogen peroxide, equal to $22.2^{\star} 10^{3} \mathrm{mM}^{-1} \mathrm{~cm}^{-1}$ and expressed in $\mathrm{nmol} / \mathrm{min} / \mathrm{mg}$ protein. ${ }^{13}$

\section{Determination of superoxide dismutase activity}

The activity of SOD was determined in the ultracentrifuged of the brain by inhibition of formazan formation of $\mathrm{n}$-nitrotetrazolium chloride (HTC). HTC was used as an indicator of $\mathrm{O}^{2 *}$ - the reduced form of which (formazan) was dissolved in acetone. To produce $\mathrm{O}^{2 *}$ - a photochemical system containing $2.8^{*} 10^{-5} \mathrm{M}$ riboflavin solution, $1^{*} 10^{-2} \mathrm{M}$ solution of tetramethylethylenediamine in $0.05 \mathrm{M} \mathrm{K}$-phosphate buffer ( $\mathrm{pH} 7.8$ ) was used for irradiation with a fluorescent lamp on $20 \mathrm{~cm}$ for 5 minutes at room temperature. To release SOD from cell organelles, a $0.5 \%$ solution of deoxycholate was used. The reaction was stopped by the addition of a $20 \%$ solution of TCA and acetone. The optical density was recorded on CPC-3 at $440 \mathrm{~nm}$. The activity of SOD in the unit of act / mg protein. ${ }^{14}$

\section{Determination of the activity of glutathione peroxidase}

The activity of glutathione peroxidase (GP) was determined in the brain ultracenterate in the conjugated glutathione reductase reaction by the loss of NADPH. The activity was recorded in a medium containing $50 \mathrm{mM} \mathrm{K}, \mathrm{Na}$-phosphate buffer, $\mathrm{pH} 7.4 ; 1.0 * 10^{-3} \mathrm{M}$ EDTA, $0.2^{\star} 10^{-3} \mathrm{M}$ $\mathrm{NADPH}, 1.0{ }^{\star} 10^{-3} \mathrm{M} \mathrm{GSH}, 1$ unit. act $/ \mathrm{ml}$ of glutathione reductase, 30-60 $\mu$ g protein per $\mathrm{ml}$ of medium on CPC-3 at $340 \mathrm{~nm}$. As a substrate in determining the total activity of glutathione peroxidase, cumene hydroperoxide was added to the medium at a concentration of $1.5^{\star} 10^{-3} \mathrm{M}$. The reaction was started by the addition of a substrate and was carried out at a temperature of $25^{\circ} \mathrm{C}^{14}$ The protein concentration was determined in the reaction with Folin reagent.

\section{Methods for assessing changes in the concentration of isoenzymes NOS and asymmetric dimethylarginine (ADMA)}

The concentration of isozymes of nitric oxide synthase (eNOS, nNOS, iNOS) was determined in rat brain homogenate by the method of enzyme immunoassay using standard Cloud Clone Corp. Reagents. Sample preparation and analysis progress closely corresponded with the instructions attached to each kit of instructions. The results were read on an Infinite F50 microplate reader (Tecan, Austria).

\section{Statistical methods}

Results of the experiments were processed by using STATISTICA 6.0 software (StatSoft, Inc., USA). The average value and the standard error of the mean value were calculated, the data were expressed as $M \pm \sigma$. The obtained results were checked for normality of the distribution using the Shapiro-Wilk test. In the case of subordination to the laws of normal distribution, of the Student $t$-test Bonferroni amendment for multiple comparisons was used to compare the means. Otherwise, further statistical processing of the experimental results was carried out using the nonparametric Mann-Whitney U -test.

\section{RESULTS}

\section{Evaluation of the antioxidant properties of 4-hydroxy-} 3,5-di-tret-butyl cinnamic acid.

Because of the study, it was established that focal cerebral ischemia leads to a shift in pro / antioxidant balance towards the formation of oxidants, which is confirmed by an increase in the concentration of MDA and DC in a group of negative control rats, compared with mice with a pseudopositive $145.7 \%(p<0.01)$ and $178.8 \%(p<0.05)$ (Table 1$)$, respectively. Also, in the NC of a group of animals against the background of cerebral ischemia, the activity of the enzymes decreased (in comparison with the PO group of rats): SOD by 20.3 times $(p<0.05)$, catalase by a $7(p<0.05)$, glutathione peroxidase - by 6.4 times $(p<0.05)$.

Oral administration of mexidol against the background of cerebral ischemia contributed to a decrease in the concentration of malonic dialdehyde and diene conjugates, relative to the $\mathrm{NC}$ of the group of rats by $70.1 \%(p<0.02)$ and $69.3 \%(p<0.02)$, respectively. Also, against the background of the introduction of mexidol, there was an increase in the activity of SOD by 19.4 times $(p<0.02)$ (Table 1$)$, catalase by $245.5 \%$ $(p<0.05)$, compared with the group of negative control rats, (Table 1), GP - by $292.8 \%(p<0.02)$.

The introduction of thioctic acid resulted in a statistically significant decrease in the concentration of MDA by $41.4 \%(p<0.05)$, as compared with the group of negative control animals, and a $90.9 \%$ increase in catalase and GP activity $(p<0.05)$ and $127.3 \%(p<0.05)$, respectively. The remaining parameters studied did not differ statistically significantly from the NC of the group of animals (Table 1 ).

The use of 4-hydroxy-3,5-di-tret-butyl cinnamic acid promoted the restoration of pro / antioxidant equilibrium in the background of focal cerebral ischemia (Table 1). This fact is confirmed by a decrease in the concentration of MDA (in comparison with the group of negative control rats) by $52.6 \%(p<0.05)$ and DC by $88.5 \%(\mathrm{p}<0.05)$. Also, against the background of the introduction of 4-hydroxy-3,5-di-tret-butyl cinnamic acid, the activity of antioxidant protection enzymes increased: SOD - 18 times $(p<0.05)$, catalase - 1456\% $(p<0.02)$ and glutathione peroxidase - in 4.1 times $(p<0.05)$. 
Table 1: Antioxydant effect of 4-hydroxy-3,5-di-tert-butyl cinnamic acid.

\begin{tabular}{cccccc}
\hline Group & PO & NC & Mexidol & Thioctic acid & ATACL \\
\hline \multirow{2}{*}{ SOD, unit of act / mg } & $356,53 \pm$ & $17,52 \pm$ & $340,68 \pm$ & $134,59 \pm$ & 315 \\
& 199,112 & $11,518 \#$ & $111,667 \Delta$ & 76,583 &, $40 \pm$ \\
& & & & & $99,966 \alpha$ \\
GP, unit of act / mg & $557,20 \pm$ & $87,28 \pm$ & $342,81 \pm$ & $198,38 \pm$ & $360,33 \pm$ \\
& 82,639 & $13,203 \#$ & $65,175 \Delta$ & $40,591 \alpha$ & $20,39 \alpha$ \\
Catalase, $\mathrm{nmol} / \mathrm{min} / \mathrm{mg}$ & $0,78 \pm$ & $0,11 \pm$ & $0,38 \pm$ & $0,21 \pm$ & $0,27 \pm$ \\
& 0,25 & $0,028 \#$ & $0,102 \alpha$ & $0,107 \alpha$ & $0,031 \Delta$ \\
MDA, $\mathrm{nmol} / \mathrm{mg}$ & $9,94 \pm$ & $24,42 \pm$ & $14,36 \pm$ & $17,26 \pm$ & $16,00 \pm$ \\
& 1,117 & $2,296^{*}$ & $1,328 \Delta$ & $2,122 \alpha$ & $1,627 \alpha$ \\
DC, $\mathrm{nmol} / \mathrm{mg}$ & $8,12 \pm$ & $22,64 \pm$ & $13,37 \pm$ & $16,98 \pm$ & $12,01 \pm$ \\
& 2,097 & $4,182^{*}$ & $4,891 \Delta$ & 6,743 & $2,982 \alpha$ \\
\hline
\end{tabular}

Note: ${ }^{*}$ - statistically significant relative to the PO group of rats $(\mathrm{p}<0.01)$;

\# - statistically significant relative to the PO group of rats $(\mathrm{p}<0.05)$;

$\Delta$ - statistically significant relative to the NC group of rats $(\mathrm{p}<0,02)$;

$\alpha$ - statistically significant relative to the NC group of rats $(\mathrm{p}<0.05)$.

Table 2: Influence of the ATACL compound on the activity of the NOS isoenzymes and a ADMA concentration.

\begin{tabular}{ccccccc}
\hline Group & PO & NC & Mexidol & $\begin{array}{c}\text { Thioctic } \\
\text { acid }\end{array}$ & Sulodexide & ATACL \\
\hline ADMA & $34,9 \pm$ & $68,4 \pm$ & $49 \pm$ & $48,3 \pm$ & $45,2 \pm$ & $37,2 \pm$ \\
$\mathrm{ng} / \mathrm{ml}$ & 0,029 & $0,052 \#$ & $0,045^{*}$ & $0,074^{*}$ & $0,041^{*}$ & $0,036^{*}$ \\
$\mathrm{nNOS}$ & $16,7 \pm$ & $27,3 \pm$ & $23,8 \pm$ & $18,4 \pm$ & $12 \pm$ & $17 \pm$ \\
$\mathrm{ng} / \mathrm{ml}$ & 0,013 & $0,045 \#$ & 0,012 & $0,056^{*}$ & $0,011^{*}$ & $0,027^{*}$ \\
$\mathrm{iNOS}$ & $13 \pm$ & $17 \pm$ & $12,2 \pm$ & $13,4 \pm$ & $10,1 \pm$ & $10,5 \pm$ \\
$\mathrm{ng} / \mathrm{ml}$ & 0,008 & $0,003 \#$ & $0,013^{*}$ & $0,006^{*}$ & $0,006^{*}$ & $0,002^{*}$ \\
$\mathrm{eNOS}$ & $77,9 \pm$ & $47,1 \pm$ & $53,6 \pm$ & $57,3 \pm$ & $60,7 \pm$ & $100,9 \pm$ \\
$\mathrm{pg} / \mathrm{ml}$ & 0,088 & $0,065 \#$ & 0,069 & 0,048 & $0,095^{*}$ & $0,022^{*}$ \\
\hline
\end{tabular}

Note: ${ }^{*}$ - statistically significant relative to the NC group of rats $(\mathrm{p}<0.05)$;

\# - statistically significant relative to the PO group of rats $(\mathrm{p}<0.05)$

Estimation of the effect of 4-hydroxy-3,5-di-tretbutyl cinnamic acid on the activity of isoenzymes of NO-synthase and the concentration of asymmetric dimethyl arginine.

In falsely operated animals, the concentration of isozymes NOS (eNOS, nNOS, iNOS) was $77.9 \pm 0.009 \mathrm{pg} / \mathrm{ml}, 13 \pm 0.008 \mathrm{ng} / \mathrm{ml}$ and $16.7 \pm$ $0.013 \mathrm{~g} / \mathrm{ml}$, respectively (Table 2 ). In this case, the concentration of ADMA in PO animals was $34.9 \pm 0.029 \mathrm{ng} / \mathrm{ml}$, respectively (Table 2).

In conditions of focal cerebral ischemia, an increase in the concentration of ADMA in comparison with the PO group of animals by $96 \%(p<0.05)$ was noted in rats. It should be noted that the increase in ADMA concentration correlated with the dissociation of NO-synthase system activity, which is manifested by an increase in the concentration of nNOS and iNOS, as well as a decrease in the concentration of eNOS in the group of negative control rats with respect to the PO group of animals by $63.5 \%$ ( $p<0.05), 30.8 \%(p<0.05)$, and 65.4\% ( $p<0.05)$, respectively (Table 2$)$.

The use of mexidol in experimental cerebral ischemia contributed to a decrease in the concentration of ADMA and iNOS in rats, $40 \%(p<0.05)$ and $39.3 \%(p<0.05)$, respectively. Against the background of the use of mexidol, the concentration of eNOS and nNOS was statistically significantly different from the similar parameters in the NC group of rats.
In animals treated with thiectic acid, under conditions of focal cerebral ischemia relative to the NC group of rats, the concentration of ADMA, nNOS and iNOS decreased by 41.6\% $(p<0.05), 48.3 \%(p<0.05)$, and $26.9 \%(p<0.05)$, respectively (Table 2 ).

Against the background of the administration of sulodexide in rats, relative to the NC of a group of animals, a decrease in the concentration of ADMA by $53.1 \%(p<0.05)$, as well as nNOS and iNOS by $127.5 \%$ $(p<0.05)$ and $68.3 \%(p<0.05)$, respectively (Table 2$)$. In addition, in rats receiving sulodexide, an increase in the concentration of eNOS was observed compared to the group of negative control animals by $28.9 \%$ $(p<0.05)$.

In rats receiving 4-hydroxy-3,5-di-tret-butyl cinnamic acid, the concentration of ADMA decreased by $83.9 \%(p<0.05)$ in relation to the group of negative control animals. Also, in rats against the background of the ATACL compound, the concentration of nNOS and iNOS decreased by $60.6 \%(p<0.05)$ and $61.9 \%(p<0.05)$, respectively (Table 2$)$. The content of eNOS in this group of animals, on the other hand, increased by $114.2 \%$ relative to the $\mathrm{NC}$ of the group of rats $(p<0.05)$.

\section{DISCUSSION}

Ischemic stroke is a formidable disease that annually takes hundreds of thousands of human lives. The number of cases of labor disability of 
the population who have undergone ischemic stroke is also terrifying, which puts the pharmacocorrection of this condition at the forefront. It is known that the effect on the endothelium of the vessels can neutralize the consequences of ischemic stroke, which predetermines its role as a possible pharmacotherapeutic "target". In this case, a positive effect on the state of the endothelial function can be realized through many mechanisms, including a direct effect on the activity of the NO-synthase system, suppression of the formation of peroxonitrite (antioxidant properties), deactivation of apoptosis, etc.

In this study, endotheliotropic activity of 4-hydroxy-3,5-di-tret-butyl cinnamic acid has been established. By restoring the activity of enzymes of endogenous antioxidant protection, the 4-hydroxy-3,5-di-tret-butyl cinnamic acid compound is able to reduce the generation of free radicals. ${ }^{16}$ Elimination from the chain of free radical transformations of active oxygen radicals leads to the termination of peroxonitrite formation and as a result, the toxic effect of reactive NO forms on vascular endotheliocytes decreases, which is accompanied by an increase in their functional activity. ${ }^{17}$ In addition, an important aspect of the endothelioprotective effect of 4-hydroxy-3,5-di-tret-butyl cinnamic acid is a direct stimulating effect on the eNOS function (as well as an increase in its activity by suppressing the formation of ADMA), which, together with a decrease in the catalytic properties of nNOS and iNOS, adequate NO production and the tone of the cerebral vessels, realizing the cerebroprotective effect. ${ }^{18}$

\section{CONCLUSION}

The endothelioprotective effect 4-hydroxy-3,5-di-tret-butyl cinnamic acid can be based on the antioxidant properties of this compound (reduce the formation of MDA and DC by $52.6 \%(p<0.05)$ and $88.5 \%$ $(p<0.05)$, respectively, and to restore the activity of SOD - 18 times $(p<0.05)$, catalase $-1456 \%(p<0.02)$ and glutathione peroxidase -4.1 $(p<0.05)$, as well as a decrease in NOS dissociation and a decrease in the formation of ADMA by $83.9 \%(p<0.05)$. According to the results of the research, further study of 4-hydroxy-3,5-di-tret-butyl cinnamic acid seems to be promising to develop an endothelioprotective agent for the treatment of cerebrovascular disorders of ischemic origin.

\section{ACKNOWLEDGEMENT}

This research received no specific grant from any funding agency in the public, commercial, or not-for-profit sectors.

\section{CONFLICT OF INTEREST}

The authors statement no conflict of interest with the submitted manuscript.

\section{ABBREVIATIONS}

NMDA: N-methyl-D - aspartate receptor; PO: pseudooperated animals; NC: negative control ATACL - 4-hydroxy-3,5-di-tret-butylcinnamic acid; DC: diene conjugates; MDA: malonic dialdehyde; SOD: superoxide dismutase; HTC: nitro blue tetrazolium; GP: glutathione peroxidase; ADMA: asymmetric dimethylarginine; iNOS: inducible synthase of nitric oxide: eNOS: endothelial synthase of nitric oxide; nNOS: neuronal synthase nitric oxide; NADPH: nicotinamidadeninedinucleotid phosphate reduced; EDTA: ethylendiaminetetraacetic; GSH: reduced glutathione.

\section{REFERENCES}

1. He X, Sandhu HK, Yang Y, Hua F, Belser N, Kim DH, et al. Neuroprotection against hypoxia/ischemia: delta-opioid receptor-mediated cellular/molecular events. CMLS. 2013;70(13):2291-303.

2. Kim JY, Kawabori M, Yenari MA. Innate inflammatory responses in stroke: mechanisms and potential therapeutic targets. Cur med chem. 2014;21(18):2076-97.

3. Chen F, Qi Z, LuoY, et al. Non-pharmaceutical therapies for stroke: Mechanisms and clinical implications. Prog. In neurobiology. 2014;10:246-69.

4. Blum A, Vaispapir V, Keinan-Boker L, Soboh S, Yehuda H, Tamir S. Endothelial Dysfunction and Procoagulant Activity in Acute Ischemic Stroke. J Vasc Interv Neurol. 2012;5(1):33-9.

5. Kaisar MA, Prasad S, Cucullo L. Protecting the BBB Endothelium against Cigarette Smoke-Induced Oxidative Stress Using Popular Antioxidants: Are they beneficial? Brain research. 2015;1627:90-100.

6. Favero G, Paganelli C, Buffoli B, Rodella LF, Rezzani R. Endothelium and Its Alterations in Cardiovascular Diseases: Life Style Intervention. Biomed Res Int 2014; 2014:801896.

7. Terpolilli NA, Moskowitz MA, Plesnila N. Nitric oxide: Considerations for the treatment of ischemic stroke. J Cereb Blood Flow Metab. 2012;32(7):1332-46.

8. Tyurenkov IN, Voronkov AV, Sliezans AA. Study of the effect of sulodexide on endothelium - dependent vasodilation of cerebral vessels in animals with streptozotocin - induced diabetes mellitus. Diab Mell. 2011;3:12-5.

9. Korokin MV, Pashin EN. The study of endothelioprotective and coronary action of 3-oxopyridine derivatives. Kuban Scien Med J. 2009;4(109):104-9.

10. Kochkarov VI, Molchanova OV, Pokrovskaya TG. Endothelioprotective action of a combination of thioctic acid with rosuvastatin with L-NAME induced deficiency of nitric oxide Scien. Bull of Belgorod State University. Series Med. Pharm. 2014;182(11):192-3.

11. Bederson JB, Pitts LH, Tsuji M, Nishimura MC, Davis $R L$, et al. Rat middle cerebral artery occlusion: evaluation of the model and development of a neurologic examination. Stroke.1986;17(3):472-6.

12. Gavrilov VB, Mishkorudnaya MI. Spectrophotometric determination of lipid hydroperoxides in blood plasma. Lab work. 1983;3:33-5.

13. Korolyuk MA. Method for the determination of catalase activity. Lab work. 1988;1:16-9.

14. Chumakov VN, Osinskaya LF. Quantitative method for determining the activity of zinc-, copper-dependent superoxide dismutase in biological material Ques. Med chem. 1977;5:712-6.

15. Pierce S, Tappel AL. Glutathione peroxidase activities from rat liver Biochim. ET biophys. Acta. 1978;523(1):27-36.

16. Rao PV, Gan SH. Cinnamon: A Multifaceted Medicinal Plant. Evid Based Complement Alternat Med.: eCAM. 2014.642942.

17. Garry PS, Ezra M, Rowland MJ, Westbrook J, Pattinson KT. The role of the nitric oxide pathway in brain injury and its treatment--from bench to bedside. Exp Neurol. 2015;263:235-43.

18. Lapchak PA. Neuroprotective and neurotrophic curcuminoids to treat stroke: a translational perspective. Expert Opin Investig Drugs. 2011;20(1):13-22.

Article History: Submission Date : 18-11-2017 ; Revised Date : 30-12-2017; Acceptance Date : 08-01-2018.

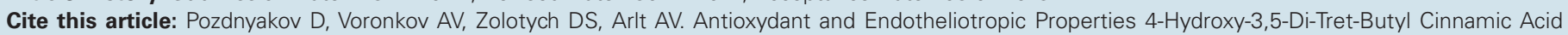
Under Conditions of Experimental Brain Ischemia. J Young Pharm. 2018;10(2):193-6. 